\title{
Imaging small-amplitude magnetization dynamics in a longitudinally magnetized microwire
}

\author{
V. V. Kruglyak, P. S. Keatley, A. Neudert, M. Delchini, and R. J. Hicken \\ School of Physics, University of Exeter, Stocker Road, Exeter EX4 4QL, United Kingdom \\ J. R. Childress and J. A. Katine \\ Hitachi Global Storage Technology, San Jose Research Center, 3403 Yerba Buena Road, San Jose, California 95135, USA
}

(Received 8 March 2007; revised manuscript received 8 April 2008; published 16 May 2008)

\begin{abstract}
We have used time-resolved scanning Kerr microscopy to study spin waves in a magnetic microwire subjected to a bias magnetic field applied parallel to its long axis. The spin-wave spectra obtained from different points near one end of the wire reveal several normal modes. We found that modes of a higher frequency occupied regions located further from the end of the wire. This was interpreted in terms of the confinement of the spin-wave modes by a nonuniform demagnetizing field. Furthermore, at a particular distance from the end of the wire, two or more modes occupying different regions along the width of the wire were observed. This was interpreted in terms of the confinement of the spin-wave modes due to an asymmetric variation in the local angle between the static magnetization and the effective direction of the wave vector of the confined modes. Images of the dynamic magnetization that are acquired at fixed pump-probe time delays revealed stripes lying perpendicular to the long axis of the wire and, hence, to the applied magnetic field. We interpret the stripe pattern in terms of a collective mode of the quasiperiodic system of ripple domains existing within the polycrystalline sample. Our results give an additional insight into the connection between the nonuniform static magnetic state in small magnetic elements and their precessional dynamics, which is fundamentally important for the design of future high-speed switching and spin-wave logic devices of magnonics.
\end{abstract}

DOI: 10.1103/PhysRevB.77.172407

PACS number(s): 75.40.Gb, 75.70.Kw, 75.30.Ds

The inherent nonuniformity of magnetization configurations in nonellipsoidal magnetic elements makes them the perfect objects for the investigation of wave phenomena in nonuniform media. The elementary excitations of magnetic media (so-called spin waves) have a strongly nonlinear dispersion, which is complicated by long-range magnetodipole interaction. In most magnetic elements of finite size, the latter remarkably makes the internal magnetic field nonuniform even when the magnetization configuration is almost uniform. The nonuniform internal field may lead to the spatial confinement of spin-wave modes ${ }^{1,2}$ while the dephasing of the modes leads to a nonuniform magnetic response to a uniform pulsed magnetic field. ${ }^{3-7}$ Apart from the fundamental interest, the studies of the magnetization dynamics in small magnetic elements are driven by the fast growing market of high-speed magnetic data storage devices. In particular, chips incorporating magnetic random access memory are now becoming available. ${ }^{8}$

Time-resolved scanning Kerr microscopy (TRSKM) ${ }^{3}$ has become one of the most widely used tools for imaging the spatial character of the magnetization dynamics within micrometer-sized magnetic disks, ${ }^{3,6}$ stripes, ${ }^{2,9}$ rectangles, ${ }^{3,10}$ and squares. ${ }^{4,5,7,11,12}$ Magnetization dynamics in micrometersized magnetic tunnel junctions were imaged in Ref. 13. Although the spatial character of the magnetization dynamics in nanometer-sized elements cannot be directly studied by TRSKM, the lack of a spatial resolution can be circumvented by combining the measurement of the time domain response from arrays of nanomagnets with thorough micromagnetic modeling. ${ }^{14-16}$

In this Brief Report, TRSKM measurements were used to investigate the magnetization dynamics near one end of a long rectangular ferromagnetic wire. By using the TRSKM as a submicrometer probe of the magnetization dynamics at different points on the wire, we recorded its time- and position-dependent responses to a pulsed magnetic field. The fast Fourier transform (FFT) spectra calculated from the signals are discussed in terms of the nonuniform demagnetizing field near the wire ends and the associated confinement of precessional modes and the variation in the local angle between the static magnetization and the effective direction of the wave vector of the confined modes. The images of the dynamic magnetization acquired at fixed pump-probe time delays revealed irregular stripes lying perpendicular to the long axis of the wire. We interpret the stripe pattern in terms of a collective mode of the quasiperiodic system of ripple domains ${ }^{17}$ existing within this polycrystalline sample.

A $\quad \mathrm{Ta}(5 \mathrm{~nm}) / \mathrm{Co}_{80} \mathrm{Fe}_{20}(4 \mathrm{~nm}) / \mathrm{Ni}_{88} \mathrm{Fe}_{12}(10.8 \mathrm{~nm}) /$ $\mathrm{Ta}(10 \mathrm{~nm})$ film was sputtered onto a $\mathrm{Si}$ substrate and patterned, by using a combination of electron beam lithography and ion milling, into a rectangular $6 \times 200 \mu \mathrm{m}^{2}$ wire. The compositions of the $\mathrm{Ni}_{88} \mathrm{Fe}_{12}$ and $\mathrm{Co}_{80} \mathrm{Fe}_{20}$ alloys were chosen so that the magnetostrictions of these two layers would partially compensate for each other, leaving the bilayer with a small net negative magnetostriction. ${ }^{14,18}$ The $\mathrm{Co}_{80} \mathrm{Fe}_{20}$ alloy composition also gave a stable fcc structure. The direction of the easy axis was set by field annealing. A series of continuous film reference samples with different relative thicknesses of $\mathrm{Co}_{80} \mathrm{Fe}_{20}$ and $\mathrm{Ni}_{88} \mathrm{Fe}_{12}$ layers were codeposited onto 1 in. diameter glass substrates. Vibrating sample magnetometry (VSM) measurements made on the reference samples showed that $1.2 \mathrm{~nm}$ of the $\mathrm{Ni}_{88} \mathrm{Fe}_{12}$ layer thickness was lost due to an interdiffusion with the Ta cap. ${ }^{14}$ To make pump-probe measurements, a transmission line structure with a $30 \mu \mathrm{m}$ track width and separation was deposited around the elements so that they experienced an out-of-plane pulsed magnetic field. The pulsed field had rise and decay times of about 70 ps and $2.2 \mathrm{~ns}$, respectively, and a maxi- 
mum strength of about $0.9 \mathrm{mT}$ and was uniform over the area of the sample to better than $1 \% .{ }^{19}$ The measurements were performed in the TRSKM configuration described in more detail elsewhere. ${ }^{15,19}$ In this configuration, the measured signal was proportional to the polar Kerr rotation and, hence, to the out-of-plane component of the magnetization under the submicron optical probe spot. A static field $\mathbf{H}$ was applied in the plane of the sample, which was parallel to the tracks of the transmission line structure and to the wire length. Each value of the static field used approached a much greater value that was sufficient to cause saturation.

The saturation magnetizations of the $\mathrm{Co}_{80} \mathrm{Fe}_{20}$ and $\mathrm{Ni}_{88} \mathrm{Fe}_{12}$ were deduced to be 1445 and $585 \mathrm{kA} / \mathrm{m}$, respectively. Since the two layers are strongly coupled by the interlayer exchange interaction, one can treat them as a single layer with a thickness equal to the sum of their individual thicknesses $(13.6 \mathrm{~nm})$ and with a magnetization given by a volume-weighted average of their saturation magnetizations $(838 \mathrm{kA} / \mathrm{m})$. Uniaxial and surface anisotropy parameters of $(950 \pm 70) \mathrm{J} / \mathrm{m}^{3}$ and $-(0.491 \pm 0.019) \mathrm{mJ} / \mathrm{m}^{2}$ were deduced from an analysis of the time-resolved measurements made upon a codeposited $10 \mu \mathrm{m}$ square element. The easy axis of the uniaxial anisotropy was found to be canted by about $4^{\circ}$ from the wire's long axis. In the analysis, the frequency of the quasiuniform precessional mode was extracted from the FFT spectrum that was calculated from the timeresolved signal measured at a particular value and orientation of the bias magnetic field. The dependence of the quasiuniform mode frequency on the value and orientation of the bias field was then fitted by using the Kittel formula in the quasialignment approximation, assuming a value of 2.1 for the $g$ factor and the value of the saturation magnetization determined from the VSM measurements. The validity of the assumption of the quasialignment was verified by performing dynamical macrospin simulations in which the static orientation of the magnetization was determined by using the steepest descent method.

The FFT spectra calculated from the measured timeresolved Kerr rotation signals with the pulsed field profile subtracted are presented in Figs. 1 and 2 for different points on the sample and for values of the bias magnetic fields of 5.5 and $15.2 \mathrm{mT}$. In the line scans parallel to the long axis of the wire (Fig. 1), we observed modes of increased frequency as the distance from the end of the wire was increased. This may be explained in terms of the nonuniform demagnetizing field near the wire end and the associated confinement of precessional modes. ${ }^{1,2}$ The demagnetizing field decreases and, hence, the total internal magnetic field averaged over the region in which precessional modes are confined increases with increasing distance from the end. In the FFT spectra calculated from the signals acquired from points $0.5 \mu \mathrm{m}$ from the wire end, several modes are visible. This is consistent with the spin-wave well picture, ${ }^{1,2}$ within which a greater number of modes are expected to reside near the bottom of the well, i.e., near the minimum of the total internal magnetic field.

As shown in Fig. 2, a variation in the mode frequency is also observed in the linear scans parallel to the short axis of the wire. Assuming that the static magnetic configuration is similar to that shown in Fig. 2(a), this can be explained in
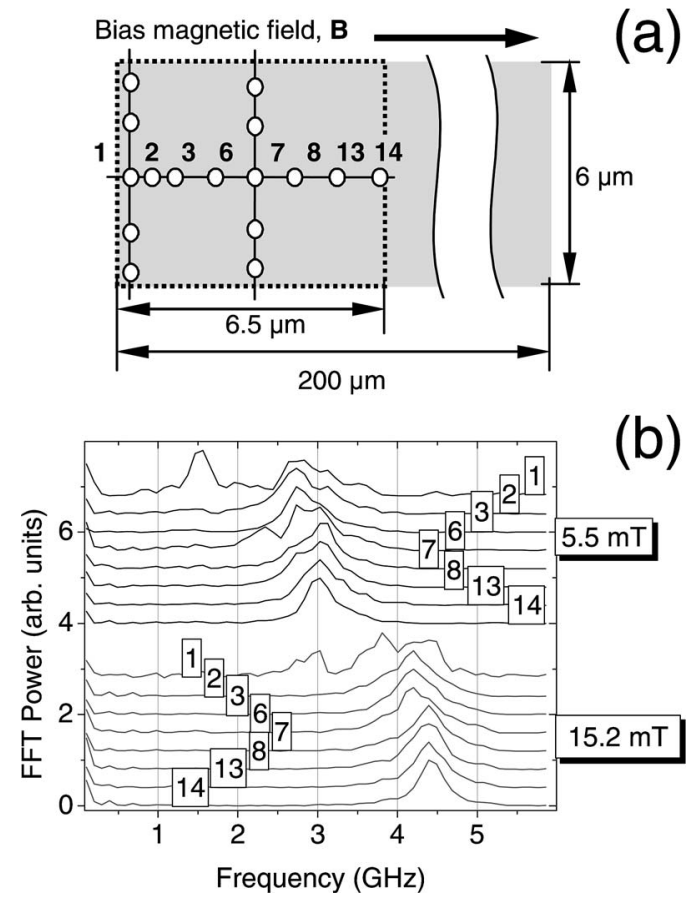

FIG. 1. (a) The geometry of the sample is shown. The white circles show the points of the wire from which the time-resolved signals were measured. The dashed rectangle shows the part of the wire from which the images were acquired. (b) The FFT power spectra calculated from the time-resolved signals acquired from the different points shown in (a) are shown for the bias field values of 5.5 and $15.2 \mathrm{mT}$. The background due to the pulsed field profile was subtracted from the time-resolved signals prior to FFT.

terms of the asymmetric variation in the local angle between the static magnetization and the effective direction of the wave vector of the confined modes. ${ }^{9,16,20}$ This variation makes a position-dependent contribution to the dynamic effective magnetic field for a precessional mode of a particular frequency and, thereby, determines the width of the region in which the mode is confined. This kind of mode confinement was not observed in the studies of wires perpendicularly magnetized to their length, ${ }^{1,2}$ where the variations in the angle between the static magnetization and the effective wave vector in the direction perpendicular to the bias magnetic field were extremely slow. The character of the variation is different at different distances from the end of the wire, depending also upon the value of the bias field. This means that the nonuniform magnetic configuration near the end is probably more complex than the simplified one shown in Fig. 2(a).

The images of the dynamic magnetization that are acquired at fixed pump-probe time delays are presented in Fig. 3. They reveal an irregular stripe pattern lying perpendicular to the long axis of the wire and, hence, to the applied magnetic field. The entire pattern oscillates in the phase and no propagation of the nonuniformity is observed. Additional images acquired from different portions of the wire showed that the nonuniform dynamics were simultaneously excited in the entire wire. In contrast to the present case of small amplitude dynamics in response to an out-of-plane pulsed field, similar patterns were also observed by Choi et al. ${ }^{10-12}$ during the 


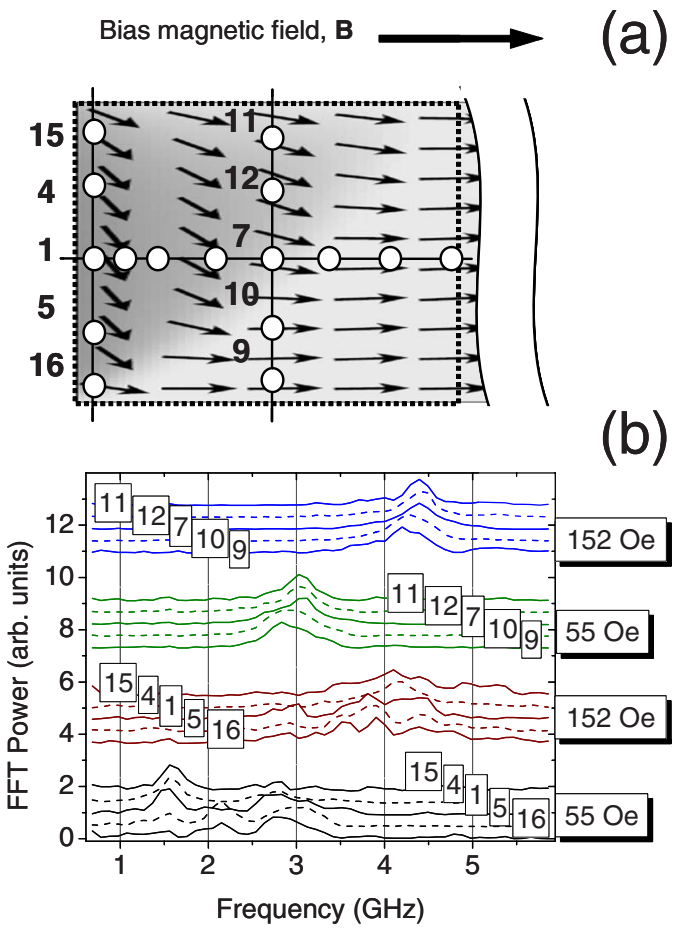

FIG. 2. (Color online) (a) The ground magnetic configuration assumed in the discussion is schematically shown. The arrows and the grayscale represent the direction of the static magnetization and the value of its in-plane component that is perpendicular to the direction of the applied field, respectively. The white circles show the points of the wire from which the time-resolved signals were measured. The dashed rectangle shows the part of the wire from which the images were acquired. (b) The FFT power spectra calculated from the time-resolved signals acquired from the different points shown in (a) are shown for the bias field values of 5.5 and $15.2 \mathrm{mT}$. The background due to the pulsed field profile was subtracted from the time-resolved signals prior to FFT. The peaks at frequencies smaller than $1 \mathrm{GHz}$ are due to an imperfect subtraction of the background and so are irrelevant to the discussion in this Brief Report.

$180^{\circ}$ magnetization reversal of micrometer-sized rectangles and squares. The pulsed field in their experiments was applied antiparallel to the initial magnetization direction, in which case, no dynamic torque was expected to act upon a uniform magnetization. However, since the static magnetization was nonuniform near the edges perpendicular to the static field, the reversal was initiated by the nonuniformity. At later time delays, the stripe pattern also formed in the central region of their samples due to a fast modulation "mediated by the exchange interaction of the precessing spins." 10 After a thorough numerical modeling, the formation of such nonequilibrium domains was attributed to the effect of thermal fluctuations, which nucleated the dynamical torque. ${ }^{10-12}$ We find this interpretation questionable since thermal fluctuations are probabilistic in nature and must be impossible to be observed by a stroboscopic technique such as TRSKM. Instead, we interpret this in terms of a collective precessional mode of the quasiperiodic system of ripple domains existing within the polycrystalline sample. ${ }^{17}$ In contrast to the thermal fluctuations, the ripple domain patterns are deterministic and

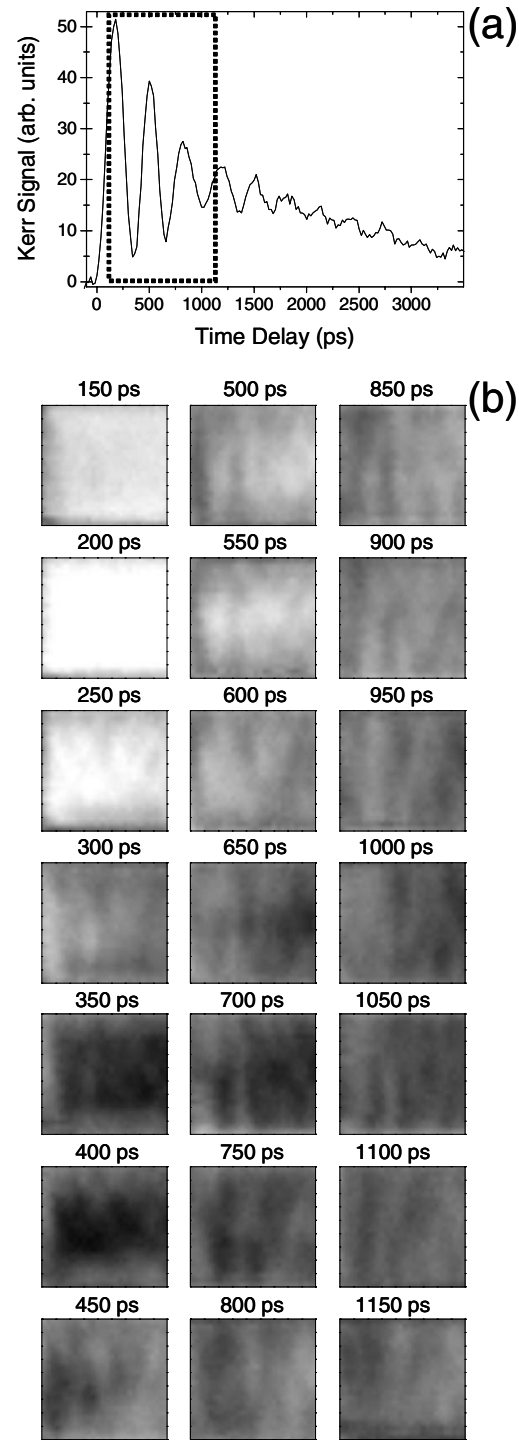

FIG. 3. (a) The time-resolved signal acquired from point 7 in Fig. 1(a) is shown for the bias field of $5.5 \mathrm{mT}$. The dashed rectangle indicates the time interval in which the images of the dynamic magnetization were acquired. (b) Grayscale images of the dynamic magnetization acquired from the $6 \times 6.5 \mu \mathrm{m}^{2}$ region near the end of the wire shown in Fig. 1(a) are presented for the indicated pumpprobe time delays. The images correspond to the same scale, which was arbitrarily chosen to give the best contrast on average. See also Ref 21.

so should be observable by a stroboscopic technique. The mode is excited, perhaps with additional help from thermal fluctuations, by a nonuniform torque due to the uniform pulsed field acting upon the nonuniform static magnetization. This interpretation is in line with the recently discovered close relationship between the spin-wave mode spectrum and the equilibrium stripe domain patterns observed in magnetic nanobars. ${ }^{22,23}$

To learn more about the static magnetic configuration in the wire, we studied the process of its magnetization reversal by means of static vector magneto-optic Kerr effect (MOKE) magnetometry ${ }^{24}$ and wide-field Kerr microscopy. ${ }^{17}$ Figures 4(a) and 4(b) present hysteresis loops acquired from point 3 


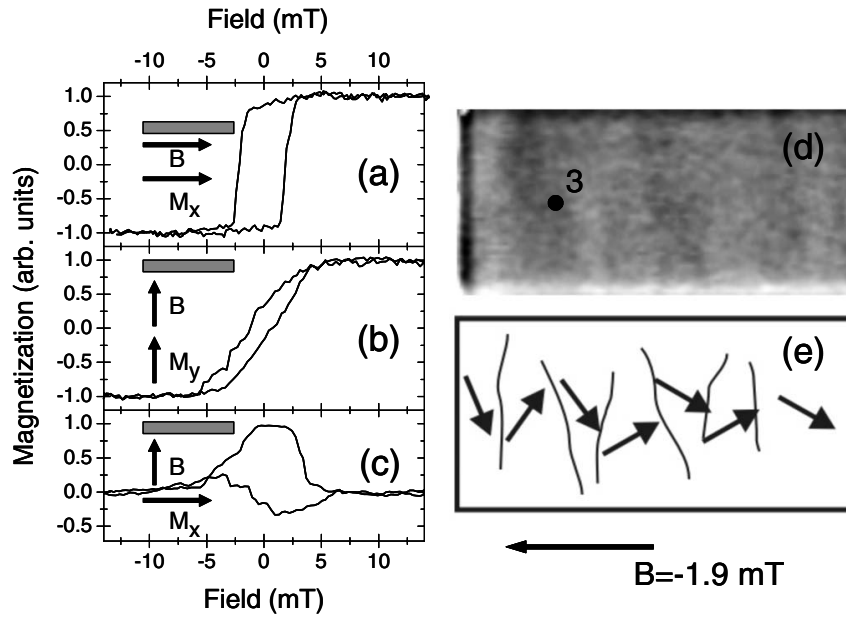

FIG. 4. [(a)-(c)] The vector MOKE hysteresis loops are shown for different orientations of the bias magnetic field relative to the wire and the direction of the sensed component of magnetization. (d) A wide-field Kerr microscopy image of the magnetic configuration in the wire is shown for the field value of $-1.9 \mathrm{mT}$ approached from saturation in the opposite (positive) direction. The magnetization component parallel to the short edge of the wire is shown. (e) A qualitative sketch of the domain structure imaged in (d) is presented.

of the wire, which is situated on its long axis $1.5 \mu \mathrm{m}$ from the end. Similar loops were acquired from other points situated at distances equal to or greater than the spot size from the edges of the wire. The loops showed a close to $100 \%$ remanence magnetization, while it is known that a magnetic field that is much stronger than that used in the reported experiments is required to fully saturate the magnetization near the edges perpendicular to the field direction..$^{20}$ In the wide-field Kerr microscopy measurements, after saturating the sample in the positive direction, the field was reduced to the value of $+5.5 \mathrm{mT}$, which is used in the dynamic studies but no nonuniformity was observed. Upon further reduction in the magnetic field and then going to the negative values, a concertinalike variation in the magnetization, such as shown in Figs. 4(d) and 4(e), became visible. The length scale of the domain pattern was similar to that observed in the dynamical studies, thus, indirectly supporting our interpretation.

In summary, we have investigated the low-amplitude precessional dynamics within and near the demagnetized region at one end of a long rectangular ferromagnetic wire. The variation in the frequency of the observed modes is governed by the local value of the internal static effective magnetic field and the local angle between the static magnetization and the effective direction of the wave vector of the confined modes. In addition, we have observed irregular stripes formed by the dynamic magnetization perpendicular to the long axis of the wire that are similar to those previously observed in the process of $180^{\circ}$ magnetization reversal. The transient stripe pattern is interpreted in terms of a collective mode of the quasiperiodic system of ripple domains, excited by a nonuniform torque due to the uniform pulsed field acting upon nonuniform static magnetization. Our results give additional insight into the connection between the nonuniform static magnetic state in the micrometer-sized elements and their high-frequency precessional dynamics. Furthermore, ferromagnetic wires are thought to be the basic building blocks for future spin-wave logic devices of magnonics. ${ }^{25}$

The authors acknowledge the financial support of the Engineering and Physical Sciences Research Council (United Kingdom) (EPSRC-GB) and the New Energy and Industrial Technology Development Organization (NEDO).
${ }^{1}$ J. Jorzick et al., Phys. Rev. Lett. 88, 047204 (2002).

${ }^{2}$ J. P. Park et al., Phys. Rev. Lett. 89, 277201 (2002).

${ }^{3}$ M. R. Freeman et al., J. Appl. Phys. 83, 6217 (1998).

${ }^{4}$ A. Barman et al., Appl. Phys. Lett. 82, 3065 (2003).

${ }^{5}$ A. Barman et al., Phys. Rev. B 69, 174426 (2004).

${ }^{6}$ C. H. Back et al., Top. Appl. Phys. 101, 137 (2006), and references therein.

${ }^{7}$ C. Bayer et al., Top. Appl. Phys. 1 01, 57 (2006), and references therein.

${ }^{8}$ B. N. Engel et al., IEEE Trans. Magn. 41, 132 (2005).

${ }^{9}$ C. Bayer et al., Phys. Rev. B 69, 134401 (2004).

${ }^{10}$ B. C. Choi et al., Phys. Rev. Lett. 95, 237211 (2005).

${ }^{11}$ B. C. Choi et al., J. Magn. 11, 61 (2006).

${ }^{12}$ B. C. Choi et al., IEEE Trans. Magn. 43, 2 (2007).

${ }^{13}$ J. H. H. Rietjens et al., Appl. Phys. Lett. 87, 172508 (2005).

${ }^{14}$ V. V. Kruglyak et al., J. Appl. Phys. 97, 10 A706 (2005).

${ }^{15}$ V. V. Kruglyak et al., Phys. Rev. B 71, 220409 (2005).

${ }^{16}$ V. V. Kruglyak et al., Phys. Rev. B 75, 024407 (2007).

${ }^{17}$ A. Hubert and R. Schäfer, Magnetic Domains: The Analysis of Magnetic Microstructures (Springer-Verlag, Berlin, 1998).

${ }^{18}$ In general, the magnetic nanoelements within magnetoelectronic devices are subject to stress. The latter is created during fabrication, e.g., due to a thermal expansion mismatch between the element and encapsulating materials, if the operating and fabri- cation temperatures are different. A small magnetostriction is important in minimizing the magnitude of the magnetic anisotropy induced by the stress. However, if some stress is unavoidable, the magnetostriction is designed so as to aid the operation of the device. In the case of recording heads, the stress induced by lapping will result-if the magnetostriction is negative-in a magnetoelastic anisotropy that is oriented parallel to the air bearing surface, which is the desirable quiescent orientation for the sensing layer. Thus, this anisotropy will assist the proper bias of the sensor instead of working against it. However, even in the latter case, a large magnetostriction will reduce the sensitivity of the sensor and should be avoided.

${ }^{19}$ P. S. Keatley et al., arXiv:0710.4926v1 (unpublished).

${ }^{20}$ R. D. McMichael and B. B. Maranville, Phys. Rev. B 74, 024424 (2006).

${ }^{21}$ See EPAPS Document No. E-PRBMDO-77-072817 for the images individually normalized so that the white and black colors correspond to the maximum and minimum of a particular image, which do not reveal any additional features. For more information on EPAPS, see http://www.aip.org/pubservs/epaps.html

${ }^{22}$ G. Leaf et al., Phys. Rev. Lett. 96, 017201 (2006).

${ }^{23}$ M. Yan et al., IEEE Trans. Magn. 42, 3204 (2006).

${ }^{24}$ P. S. Keatley et al., J. Magn. Magn. Mater. 306, 298 (2006).

${ }^{25}$ S. V. Vasiliev et al., J. Appl. Phys. 101, 113919 (2007). 